\title{
Strong coupling theory for driven tunneling and vibrational relaxation
}

\author{
M. Thorwart ${ }^{1}$, M. Grifoni ${ }^{2,3}$, and P. Hänggi ${ }^{1}$ \\ ${ }^{1}$ Institut für Physik, Universität Augsburg, Universitätsstraße 1, D-86135 Augsburg, Germany \\ ${ }^{2}$ Institut für Theoretische Festkörperphysik, Universität Karlsruhe, D-76128 Karlsruhe, Germany \\ ${ }^{3}$ Dipartimento di Fisica, INFM, Via Dodecaneso 33, I-16146, Genova, Italy
}

(Date: August 22, 2018)

\begin{abstract}
We investigate on a unified basis tunneling and vibrational relaxation in driven dissipative multistable systems described by their $N$ lowest lying unperturbed levels. By use of the discrete variable representation we derive a set of coupled non-Markovian master equations. We present analytical treatments that describe the dynamics in the regime of strong system-bath coupling. Our findings are corroborated by "ab-initio" real-time path integral calculations.
\end{abstract}

PACS: 05.30.-d, 05.40.-a, 33.80.Be, 82.20.Pm

Dissipative tunneling in bistable systems finds widespread applications in many physical and chemical contexts. Usually, the dynamics is described by restricting a double-well potential to its lowest tunneling doublet of energy eigenstates [1]. This two-level system (2LS) describes well the dynamics at low temperature, but becomes increasingly invalid at higher temperatures, when higher lying doublets are populated. Then both tunneling between the metastable quantum wells and vibrational relaxation within the wells mix the dynamics. In the presence of an external field, e.g., a laser, the different doublets are additionally coupled. Such dissipative multilevel systems occur in the context of tunneling of magnetization in organic high-spin molecules, i.e., $\mathrm{Mn}_{12}$ [2] and $\mathrm{Fe}_{8}$ [3]. Moreover, they mimic tunneling of defects in metals [4] and hydrogen pair transfer in benzoic acid [b]. Also, resonant tunneling of the magnetic flux in SQUIDS exhibits a multilevel structure [6]. An example, where a picosecond laser driving accelerates intramolecular isomerization in malonaldehyde, is given in [7]. There, a pumping mechanism for fast hydrogen transfer ("hydrogen subway") is proposed (see also [8]). This complex driven dissipative multilevel system has been investigated numerically in [7.97 within a weak coupling approach, and in 10] within the real-time path integral tensor multiplication scheme 11]. In absence of driving, the dissipative bistable system has been researched by a numerical reactive flux analytic continuation scheme in [12]. The only analytical approach previous to our work has been attempted in [8]. In these works, however, the dynamics restricted to the two lowest tunneling doublets was investigated in the weak coupling regime. Because of its complexity, even the nondriven multistate dynamics has barely been studied [13,14]. Common to all prior works [8,13,14] is that the position operator is incorrectly assumed to be diagonal in the localized (spin) representation. This, however, holds true only when the lowest tunneling-doublet rules the dynamics, cf. (11) below. Moreover, the harmonic approximation for the two wells proposed in [13] can be justified only for a large bar- rier height where a semiclassical description is already applicable 115. Furthermore, the research in [14] is not completely based on a full microscopic Hamiltonian.

The prime objective of this work is the development of a first consistent treatment wherein tunneling (TU), driving, and vibrational relaxation (VR) are treated analytically on a common footing, beyond a Redfield-approach, and for an arbitrayry number $N$ of levels. A unified, consistent treatment becomes possible only if one uses the so termed discrete variable representation (DVR) [16]. It is in this DVR basis that the position operator is diagonal. A real-time path integral approach is used to derive a set of coupled generalized master equations for the combined VR and TU dynamics. The predictions of these equations well agree with precise numerical quantum simulations. Analytical results for the rate matrix of a $N$-level system are obtained.

The model.- To start, we consider the Hamiltonian $H(t)=H_{0}+H_{\text {ext }}(t)+H_{\mathrm{B}}$ which accounts for quantum dissipation and external, generally time-dependent control fields. The term $H_{0}=\frac{p^{2}}{2 M}+V(q)$ denotes the Hamiltonian of the isolated system. We consider a particle of mass $M$ moving in the bistable quartic potential $V(q)=\left(M^{2} \omega_{0}^{4} / 64 E_{B}\right) q^{4}-\left(M \omega_{0}^{2} / 4\right) q^{2}$, where $E_{B}$ is the barrier height, and $\omega_{0}$ the frequency of classical oscillations around the potential minima at $\pm q_{0} / 2$. For the isolated system the energy spectrum follows from the Schrödinger equation $H_{0}|n\rangle=E_{n}|n\rangle, n=1,2, \ldots$ For energies well below the barrier, the spectrum consists of a ladder of doublets. To illustrate the method, we consider the case in which only two doublets $\hbar \Delta_{1}=E_{2}-E_{1}$, and $\hbar \Delta_{2}=E_{4}-E_{3}$ contribute significantly to the dynamics. They are separated by the energy gap $\hbar \bar{\omega}_{0}=$ $\frac{1}{2}\left(E_{4}+E_{3}\right)-\frac{1}{2}\left(E_{2}+E_{1}\right) \gg \hbar \Delta_{i}$. We consider then the reduction $H_{0} \rightarrow H_{4 \mathrm{LS}}$, with $H_{4 \mathrm{LS}}$ being the Hamiltonian for the isolated four-level system (4LS). The external field is characterized by $H_{\text {ext }}(t)=-s(t) q$, with $s(t)$ being a time-dependent field. In the basis of the vectors $\left|R_{1}\right\rangle=\frac{1}{\sqrt{2}}(|1\rangle+|2\rangle),\left|L_{1}\right\rangle=\frac{1}{\sqrt{2}}(|1\rangle-|2\rangle)$, and $\left|R_{2}\right\rangle=\frac{1}{\sqrt{2}}(|3\rangle+|4\rangle),\left|L_{2}\right\rangle=\frac{1}{\sqrt{2}}(|3\rangle-|4\rangle)$, with $\left|L_{i}\right\rangle\left(\left|R_{i}\right\rangle\right)$ 
localized in the left (right) well, the discrete position operator of the system reads

$$
\begin{aligned}
q^{l o c}= & \sum_{i, j=1,2} a_{i j}\left(\left|R_{i}\right\rangle\left\langle R_{j}|-| L_{i}\right\rangle\left\langle L_{j}\right|\right) \\
& +b\left(\left|L_{1}\right\rangle\left\langle R_{2}|+| R_{2}\right\rangle\left\langle L_{1}\right|\right. \\
& \left.-\left|R_{1}\right\rangle\left\langle L_{2}|-| L_{2}\right\rangle\left\langle R_{1}\right|\right),
\end{aligned}
$$

with $a_{11}=\langle 1|q| 2\rangle, \quad a_{22}=\langle 3|q| 4\rangle, \quad a_{12}=a_{21}=$ $(\langle 1|q| 4\rangle+\langle 2|q| 3\rangle) / 2$, and $b=(\langle 1|q| 4\rangle-\langle 2|q| 3\rangle) / 2 \ll a_{i j}$. Note that, in clear contrast to the 2LS case, it is nondiagonal. For the following analytical treatment, we set $b=0$. In all results shown in the figures, however, we use $b \neq 0$. Finally, we model quantum dissipation by an ensemble of harmonic oscillators that are bilinearly coupled to the system [15], i.e., $H_{\mathrm{B}}=\frac{1}{2} \sum_{i}\left[\frac{p_{i}^{2}}{m_{i}}+m_{i} \omega_{i}^{2}\left(x_{i}-\frac{c_{i}}{m_{i} \omega_{i}^{2}} q\right)^{2}\right]$, with $J(\omega)=(\pi / 2) \sum_{i} c_{i}^{2} /\left(m_{i} \omega_{i}\right) \delta\left(\omega-\omega_{i}\right)$ being the spectral density. We assume Ohmic dissipation with an exponential cut-off $\omega_{c} \gg \omega_{0}$ and a viscous friction strength $\gamma$, such that $J(\omega)=M \gamma \omega e^{-\omega / \omega_{c}}$. We wish to evaluate the probability $P_{\mathrm{L}}(t):=\sum_{i}\left\langle L_{i}|\rho(t)| L_{i}\right\rangle$ to find at time $t>t_{0}$ the system in the left well. Here $\rho(t)$ denotes the reduced density matrix (RDM) of the system. We belabor the case in which the particle initially is prepared in the lower left state: $\rho\left(t_{0}\right)=\left|L_{1}\right\rangle\left\langle L_{1}\right|$, with the bath in thermal equilibrium at temperature $T=\left(k_{B} \beta\right)^{-1}$.

DVR description of vibrational relaxation and tunneling.-

For a harmonic bath, path integral techniques allow one to trace out analytically the bath degrees of freedom in the eigenbasis of the position operator $q$ (DVR basis). Let us start with the introduction of the DVR vectors:

$\left|\alpha_{1}\right\rangle=v\left(\left|L_{1}\right\rangle-u\left|L_{2}\right\rangle\right), \quad\left|\beta_{1}\right\rangle=v\left(\left|R_{1}\right\rangle-u\left|R_{2}\right\rangle\right)$,

$\left|\alpha_{2}\right\rangle=v\left(u\left|L_{1}\right\rangle+\left|L_{2}\right\rangle\right), \quad\left|\beta_{2}\right\rangle=v\left(u\left|R_{1}\right\rangle+\left|R_{2}\right\rangle\right)$,

with $\left|\alpha_{i}\right\rangle\left(\left|\beta_{i}\right\rangle\right)$ being localized in the left (right) well, respectively. Here, $v=1 / \sqrt{1+u^{2}}$ and $u=\left(a_{11}+\right.$ $\left.\lambda_{\alpha_{1}}\right) / a_{12}=-\left(a_{22}+\lambda_{\alpha_{2}}\right) / a_{12}$, and $\lambda_{\alpha_{i}}=-\lambda_{\beta_{i}}$ denote the position eigenvalues: $\lambda_{\alpha_{1,2}}=\left[-\left(a_{11}+a_{22}\right) \mp\right.$ $\left.\sqrt{\left(a_{11}-a_{22}\right)^{2}+4 a_{12}^{2}}\right] / 2$. Upon the introduction of the DVR tunneling matrix elements

$$
\begin{aligned}
& \Delta_{\alpha_{1} \beta_{1}} \equiv v^{2}\left(\Delta_{1}+u^{2} \Delta_{2}\right), \Delta_{\alpha_{2} \beta_{2}} \equiv v^{2}\left(u^{2} \Delta_{1}+\Delta_{2}\right), \\
& \Delta_{\alpha_{1} \beta_{2}}=\Delta_{\alpha_{2} \beta_{1}} \equiv v^{2} u\left(\Delta_{1}-\Delta_{2}\right),
\end{aligned}
$$

being a linear combination of the tunneling splittings $\Delta_{1}$ and $\Delta_{2}$, the 4 LS Hamiltonian reads in this DVR basis

$$
\begin{aligned}
& H_{4 \mathrm{LS}}^{D V R}=-\sum_{i, j=1,2} \frac{1}{2} \hbar \Delta_{\alpha_{i} \beta_{j}}\left(\left|\alpha_{i}\right\rangle\left\langle\beta_{j}|+| \beta_{j}\right\rangle\left\langle\alpha_{i}\right|\right) \\
& +\sum_{i=1,2}\left(F_{\alpha_{i}}\left|\alpha_{i}\right\rangle\left\langle\alpha_{i}\left|+F_{\beta_{i}}\right| \beta_{i}\right\rangle\left\langle\beta_{i}\right|\right)-v^{2} u \hbar \bar{\omega}_{0} R,
\end{aligned}
$$

with $F_{\alpha_{1}}=F_{\beta_{1}}=u^{2} v^{2} \hbar \bar{\omega}_{0}, F_{\alpha_{2}}=F_{\beta_{2}}=v^{2} \hbar \bar{\omega}_{0}$. The matrix $R=\left|\alpha_{1}\right\rangle\left\langle\alpha_{2}|+| \alpha_{2}\right\rangle\left\langle\alpha_{1}|+| \beta_{1}\right\rangle\left\langle\beta_{2}|+| \beta_{2}\right\rangle\left\langle\beta_{1}\right|$ accounts for intrawell transitions. It is suggestive to introduce the DVR intrawell transition elements

$$
\Delta_{\alpha_{1} \alpha_{2}}=\Delta_{\alpha_{2} \alpha_{1}}=\Delta_{\beta_{1} \beta_{2}}=\Delta_{\beta_{2} \beta_{1}} \equiv 2 v^{2} u \bar{\omega}_{0} .
$$

Because $\Delta_{\alpha_{1} \beta_{2}} \approx \Delta_{\alpha_{1} \beta_{1}} \leq \Delta_{\alpha_{2} \beta_{2}}<2 v^{2} u \bar{\omega}_{0}$, different time scales determine this VR assisted tunneling dynamics. For the left well population, one finds $P_{\mathrm{L}}(t)=$ $\sum_{i}\left\langle\alpha_{i}|\rho(t)| \alpha_{i}\right\rangle$, with the initial RDM $\rho\left(t_{0}\right)=\left|L_{1}\right\rangle\left\langle L_{1}\right|=$ $v\left(\left|\alpha_{1}\right\rangle\left\langle\alpha_{1}\left|+u^{2}\right| \alpha_{2}\right\rangle\left\langle\alpha_{2}|+u| \alpha_{1}\right\rangle\left\langle\alpha_{2}|+u| \alpha_{2}\right\rangle\left\langle\alpha_{1}\right|\right)$ being nondiagonal. By use of the notation $\mu=\alpha_{1}, \alpha_{2}, \beta_{1}, \beta_{2}$ for the index of the DVR states, the formally exact path integral expression for the diagonal elements of the RDM in the DVR basis reads $\left(\rho_{\mu \nu}:=\langle\mu|\rho(t)| \nu\rangle\right)$

$$
\rho_{\nu \nu}(t)=\sum_{\mu_{0} \nu_{0}} \int \mathcal{D} q \int \mathcal{D} q^{\prime} \mathcal{A}[q] \mathcal{A}^{*}\left[q^{\prime}\right] \mathcal{F}\left[q, q^{\prime}\right] \rho_{\mu_{0} \nu_{0}}\left(t_{0}\right),
$$

with the paths subject to the constraint $q(t)=q^{\prime}(t)=$ $\lambda_{\nu}$, and $q\left(t_{0}\right)=\lambda_{\mu_{0}}, q^{\prime}\left(t_{0}\right)=\lambda_{\nu_{0}}$, with $\left\{\lambda_{\nu}\right\}$ being the position operator eigenvalues. $\mathcal{A}[q]$ is the path weight in the absence of dissipative forces, while the influence functional $\mathcal{F}\left[q, q^{\prime}\right]$ accounts for the bath effects.

$N$-level dynamics.- Although derived for a 4LS, these equations hold for a finite number $N$ of levels. We switch to center of mass $\eta(s)=\left[q(s)+q^{\prime}(s)\right]$ and relative coordinates $\xi(s)=\left[q(s)-q^{\prime}(s)\right]$. Then, the double path integral over the " $N$-state" paths $q(s)$ or $q^{\prime}(s)$ is expressed as a single path integral over the $N^{2}$ states of the RDM in the $\left(q, q^{\prime}\right)$ plane. The case of the $4 \mathrm{LS}$ is depicted in Fig. 1. Any such path can be described as a sequence of time intervals spent in a diagonal state of the RDM ("sojourns") and time intervals between two successive visits of the diagonal states ("clusters"). The functional $\mathcal{F}$ couples different path segments. For a path with $n$ transitions at times $t_{1}, . ., t_{n}$, we introduce the cumulative off-diagonal charge $p_{j}=\sum_{i=1}^{j \leq n} \xi_{i}$, associated to the path derivative $\dot{\xi}(s):=\sum_{i=1}^{n} \xi_{i} \delta\left(s-t_{i}\right)$. With $\xi_{i}=\left(\lambda_{\mu_{i}}-\lambda_{\nu_{i}}\right)-\left(\lambda_{\mu_{i-1}}-\lambda_{\nu_{i-1}}\right)$, it yields $p_{n}=0$ within each cluster of $n$ jumps. Thus, the clusters are "neutral" objects, being only weakly interacting at high temperature and/or large Ohmic damping, due to the short range of the intercluster interaction. This suggests a generalization of the noninteracting-cluster-approximation (NICA) scheme in 17] to our driven situation, with combined VR and TU transitions also among non-nearest neighbors and with an initially nondiagonal RDM. We call this the VRTU-NICA. It yields the set of coupled generalized master equations (GME)

$\dot{\rho}_{\nu \nu}\left(t, t_{0}\right)=I_{\nu}\left(t, t_{0}\right)+\sum_{\mu=1}^{N} \int_{t_{0}}^{t} d t^{\prime} H_{\nu \mu}\left(t, t^{\prime}\right) \rho_{\mu \mu}\left(t^{\prime}, t_{0}\right)$,

where all intercluster as well as non-nearest-neighbors sojourn-cluster interactions are neglected, while keeping fully the intracluster interactions. The VRTU-NICA is expected to yield reliable results as long as the friction 
strength does not exceed level broadening among neighboring doublets or the temperature is not too low. For the case of a single tunneling doublet the DVR basis coincides with the localized basis. Then, the VRTU-NICA reduces to the familiar (driven) noninteracting-blip approximation for a $2 \mathrm{LS}$ [1]. If more than two levels are involved, the functions $H_{\nu \mu}$ and $I_{\nu}$ are expressed as power series in the DVR-Hamilton matrix elements $\Delta_{\mu \nu}$.

Sequential dynamics.- To lowest order one finds

$$
\begin{aligned}
H_{\nu \mu}\left(t, t^{\prime}\right) & =\frac{\Delta_{\nu \mu}^{2}}{2} e^{-Q_{\nu \mu}^{\prime}\left(t-t^{\prime}\right)} \cos \left[\varphi_{\nu \mu}\left(t, t^{\prime}\right)-Q_{\nu \mu}^{\prime \prime}\left(t-t^{\prime}\right)\right], \\
I_{\nu}\left(t, t_{0}\right) & =\left(\delta_{\nu \alpha_{2}}-\delta_{\nu \alpha_{1}}\right) \operatorname{Re} \rho_{\alpha_{1} \alpha_{2}}\left(t_{0}\right) \Delta_{\alpha_{1} \alpha_{2}} e^{-Q_{\alpha_{1} \alpha_{2}}^{\prime}\left(t-t_{0}\right)} \\
& \times \sin \left[\varphi_{\alpha_{1} \alpha_{2}}\left(t, t_{0}\right)-Q_{\alpha_{1} \alpha_{2}}^{\prime \prime}\left(t-t_{0}\right)\right], \nu \neq \mu .
\end{aligned}
$$

The conservation of probability yields for the diagonal elements $H_{\mu \mu}=-\sum_{\nu \neq \mu} H_{\nu \mu}$. The driving influence is captured by $\varphi_{\nu \mu}\left(t, t^{\prime}\right)=\int_{t^{\prime}}^{t} d t^{\prime \prime}\left[\varepsilon_{\mu}\left(t^{\prime \prime}\right)-\varepsilon_{\nu}\left(t^{\prime \prime}\right)\right]$, where $\varepsilon_{\nu}(t)=F_{\nu}-\lambda_{\nu} s(t)$ with $F_{\nu}$ given below (雨). Finally, the bath influence is encapsulated in the correlation functions $Q_{\mu \nu}=Q_{\mu \nu}^{\prime}+i Q_{\mu \nu}^{\prime \prime}$ with $Q_{\mu \nu}=\left(\lambda_{\mu}-\lambda_{\nu}\right)^{2} Q(t)$, and $Q(t)=\frac{1}{\hbar \pi} \int_{0}^{\infty} d \omega\left[J(\omega) / \omega^{2}\right]\left(\left\{\cosh (\beta \hbar \omega / 2)-\cosh \left[\omega\left(\frac{\hbar \beta}{2}-\right.\right.\right.\right.$ $i t)]\} /[\sinh (\beta \hbar \omega / 2)])$. Hence, to each transition an effective friction strength

$$
\alpha_{j} \equiv\left(\xi_{j} / q_{0}\right)^{2} \alpha, \quad \text { with } \quad \alpha=M \gamma q_{0}^{2} /(2 \pi \hbar),
$$

is associated. The prediction for the population $P_{\mathrm{L}}(t)$ of the left well is depicted in the inset of Fig. 2, together with the results of the quasiadiabatic propagator path integral method (QUAPI) [11] adapted to the 4LS case. For the chosen parameters, higher order coherent paths yield only minor corrections. The inset also shows that the dynamics described by (6) is well approximated by a Markovian master equation, being independent of the initial off-diagonal preparation, i.e., $\dot{\rho}_{\nu \nu}(t)=$ $\sum_{\mu} \Gamma_{\nu \mu}(t) \rho_{\mu \mu}(t)$, where $\Gamma_{\nu \mu}(t)=\int_{0}^{\infty} d t^{\prime} H_{\nu \mu}\left(t, t-t^{\prime}\right)$. The explicit time dependence of $\Gamma_{\nu \mu}$ reflects the time dependent external forcing. It is appearent that the dynamics is in this regime governed by a single exponential decay. To extract this decay rate we observe that for high-frequency fields (such as an interdoublet resonant field), averaging over a driving period is appropriate. This yields the time-independent rate-matrix

$$
\begin{aligned}
\Gamma_{\nu \mu}^{\mathrm{av}} & =\frac{\Delta_{\nu \mu}^{2}}{2} \int_{0}^{\infty} d \tau \exp \left[-Q_{\nu \mu}^{\prime}(\tau)\right] J_{0}\left(\zeta_{\mu \nu} \frac{2 s}{\Omega} \sin \left(\frac{\Omega \tau}{2}\right)\right) \\
& \times \cos \left[\left(F_{\mu}-F_{\nu}\right) \tau-Q_{\nu \mu}^{\prime \prime}(\tau)\right], \quad \nu \neq \mu
\end{aligned}
$$

where $\zeta_{\mu \nu}=\lambda_{\mu}-\lambda_{\nu}$, with $J_{0}(x)$ being the zeroth Bessel function. The main part of Fig. 2 shows the $4 \mathrm{LS}$ averaged decay rate $\Gamma^{\text {av }}$, being the smallest nonzero eigenvalue of the Markovian rate matrix $\Gamma_{\nu \mu}^{\mathrm{av}}$, vs the amplitude $s$ of a resonant $\left(\Omega=\bar{\omega}_{0}\right)$ driving field $s(t)=s \sin (\Omega t)$. Note the perfect agreement between the GME predictions and those of the QUAPI, together with the characteristic nonmonotonic behavior. The important issue of the contribution of the higher lying states is investigated in Fig. 3, where the undriven $(s=0)$ decay rate is depicted vs the number $N$ of DVR states used to truncate the full double-well problem. It is clearly seen that, for moderate friction, obeying $\Delta_{i}<\gamma<\bar{\omega}_{0}$, the truncation to the lowest two doublets is adequate even at moderate temperature $k_{B} T=0.1 \hbar \omega_{0}$. This condition implies that neighboring doublets do not overlap due to frictional broadening. Clearly, the convergence is expected to improve as the temperature is lowered. We find (not shown) that a truncation to a $4 \mathrm{LS}$ is adequate for low temperatures and slow driving fields $\left(\Omega<0.1 \omega_{0}\right)$. For highfrequency fields also higher lying states are involved in the dynamics as depicted in the inset of Fig. 3. Hence, a reduction of the driven double-well problem to a $4 \mathrm{LS}$ is problematic in the presence of a strong resonant field and moderate temperatures.

Beyond sequential dynamics.- Upon increasing the temperature $\left(k_{B} T \gtrsim \hbar \omega_{0}\right)$, a truncation to a few levels only starts to be inadequate. Because the effective friction strengths $\alpha_{j}$ scale quadratically with $\xi_{j}$, upon increasing the number $N$ of DVR states involved, the NLS effectively flows to weak coupling. For small effective $\alpha_{j}$, however, the noise action does not suppress long intervals in the off-diagonal states, and the higher order paths start to contribute. For $\gamma<0.1 \omega_{0}$, $k_{B} T \gtrsim \hbar \omega_{0}$ and $\omega_{c} \gg k_{B} T / \hbar, \omega_{0}$, we can approximate $Q(t) \approx 2 \alpha\left[\pi|t| / \hbar \beta+\ln \left(\hbar \beta \omega_{c} / 2 \pi\right)\right]+i \pi \alpha \operatorname{sgn} t$. Now the intercluster correlations cancel out exactly. The corresponding averaged Markovian rates read

$$
\begin{aligned}
\Gamma_{\nu \mu}^{\mathrm{av}}= & \sum_{n=2}^{\infty} \sum_{\left\{\mu_{j} \nu_{j}\right\}} \prod_{j=1}^{n}(-1)^{\delta_{j}}\left(\frac{i}{2}\right)^{n} \tilde{\Delta}_{\mu_{j} \nu_{j}, \mu_{j-1} \nu_{j-1}}\left(\frac{2 \pi}{\beta \hbar \omega_{c}}\right)^{\alpha_{j}} \\
& \times e^{-i \pi(-1)^{\delta_{j}} \alpha_{j} p_{j} / \xi_{j}} f_{j}, \\
f_{j}= & \int_{0}^{\infty} d \tau e^{-\frac{2 \pi \alpha_{j} p_{j}^{2}}{\hbar \beta \xi_{j}^{2}} \tau} J_{0}\left(p_{j} \frac{2 s}{\Omega} \sin \left(\frac{\Omega \tau}{2}\right)\right) e^{-i\left(F_{\mu_{j}}-F_{\nu_{j}}\right) \tau},
\end{aligned}
$$

where $p_{j}=\sum_{i=1}^{j \leq n} \xi_{i}, \delta_{j}=0$ (1) for a vertical (horizontal) jump and $\tilde{\Delta}_{\mu_{j} \nu_{j}, \mu_{j-1} \nu_{j-1}}$ is the DVR-Hamilton matrix element for the transition from $\left(\mu_{j-1}, \nu_{j-1}\right)$ to $\left(\mu_{j}, \nu_{j}\right)$, e.g. for a horizontal jump, $\tilde{\Delta}_{\mu_{j} \nu_{j}, \mu_{j-1} \nu_{j-1}}:=\Delta_{\nu_{j} \nu_{j-1}}$. Since (10) describes well the high temperature dynamics, this rate matrix with $s=0$ constitutes the starting point for an evaluation of the crossover to the classical regime. With $s \neq 0$, however, a chaotic dynamics may occur.

Conclusions.- We put forward a real-time path integral approach to investigate the interplay between vibrational relaxation and tunneling in driven, dissipative multilevel quantum systems. We succeeded in deriving a novel GME within the DVR basis which treats tunneling and intrawell dynamics on a common basis: Its Markovian approximation in (9) and (10) yields novel analytical results that well agree with those of the full 
GME. In contrast to semiclassical imaginary-time rate calculations [15], we are not limited by the requirement of thermal equilibrium at adiabatically varying external fields. Hence, our results provide a powerful tool to investigate the crossover from a quantum to a classical dynamics beyond the restrictions of the semiclassical approximation. Our choice of parameter finely mimics the situation of a picosecond laser which accelerates isomerization in malonaldehyde [7]. There a barrier height of $E_{B} \approx 1.7$ is given, to be contrasted with $E_{B}=1.4$ here. Also $\mathrm{Mn}_{12}$ and $\mathrm{Fe}_{8}$ nanomagnets, which have a spin ground state of $S=10(N=21$ levels $)$, provide an interesting example where to apply our theory [2].

We acknowledge support by the Deutsche Forschungsgemeinschaft (HA1517/14-3, P.H.), the Graduiertenkolleg GRK 283 (M.T.), and the European Community (M.G.). We thank I. Goychuk, G. Ingold, P. Reimann, and G. Schön for fruitful remarks.

[1] M. Grifoni and P. Hänggi, Phys. Rep. 304, 219 (1998).

[2] W. Wernsdorfer et al., Phys. Rev. Lett. 82, 3903 (1999); W. Wernsdorfer and R. Sessoli, Science 284, 133 (1999).

[3] C. Sangregorio et al., Phys. Rev. Lett. 78, 4645 (1997).

[4] R. I. Cukier, M. Morillo, K. Chun, and N. O. Birge, Phys. Rev. B 51, 13767 (1995).

[5] A. Stöckli et al., J. Chem. Phys. 93, 1502 (1990).

[6] R. Rouse, S. Han, and J. E. Lukens, Phys. Rev. Lett. 75, 1614 (1995).

[7] H. Naundorf, K. Sundermann, and O. Kühn, Chem. Phys. 240, 163 (1999).

[8] M. Morillo, C. Denk, and R. I. Cukier, Chem. Phys. 212, 157 (1996); R. I. Cukier, C. Denk, and M. Morillo, Chem. Phys. 217, 179 (1997).

[9] S. Kohler, R. Utermann, P. Hänggi, and Th. Dittrich, Phys. Rev. E 58, 7219 (1998).

[10] M. Thorwart and P. Jung, Phys. Rev. Lett. 78, 2503 (1997); M. Thorwart, P. Reimann, P. Jung, and R. F. Fox, Chem. Phys. 235, 61 (1998).

[11] D. E. Makarov and N. Makri, Chem. Phys. Lett. 221, 482 (1994).

[12] E. Rabani, G. Krilov, and B. J. Berne, J. Chem. Phys. 112, 2605 (2000).

[13] H. Dekker, Phys. Rev. A, 44, 2314 (1991).

[14] P. E. Parris and R. Silbey, J. Chem. Phys. 83, 5619, (1985).

[15] U. Weiss, Quantum Dissipative Systems, (World Scientific, Singapore, 1999), 2nd ed.; P. Hänggi, P. Talkner, and M. Borkovec, Rev. Mod. Phys. 62, 251 (1990).

[16] D. O. Harris, G. G. Engerholm, and W. D. Gwinn, J. Chem. Phys. 43, 1515 (1965).

[17] R. Egger, C. H. Mak, and U. Weiss, Phys. Rev. E 50, R655 (1994).

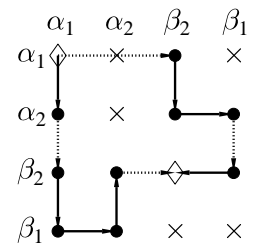

FIG. 1. The sixteen states of the reduced density matrix of a four-level system (4LS) in the discrete variable representation. Shown are two typical paths starting and ending in the diagonal states $\diamond$ Solid lines indicate vibrational relaxation transitions, dotted lines tunneling events.

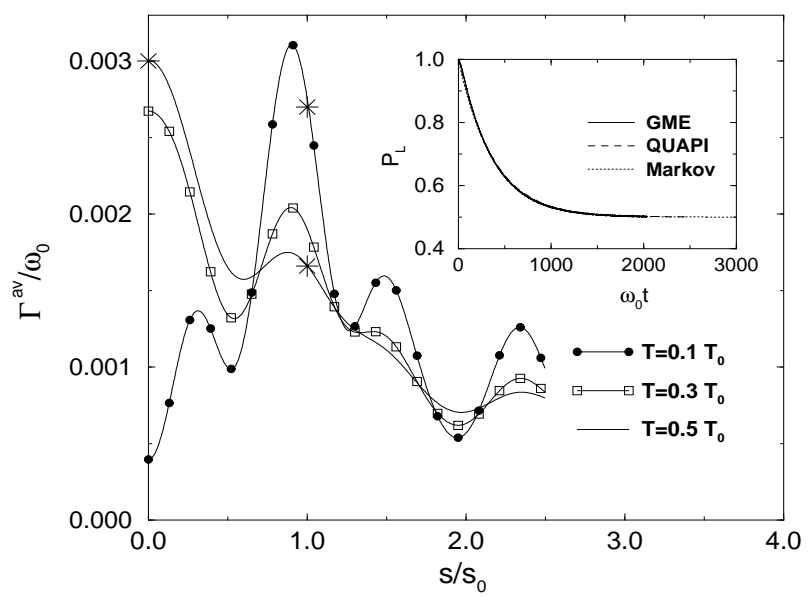

FIG. 2. Averaged transfer rate of a $4 \mathrm{LS}$ vs. the scaled $\left(s_{0}=\hbar \omega_{0} / q_{0}, q_{0}=\sqrt{\hbar / M \omega_{0}}\right)$ driving amplitude of a resonant $\left(\Omega=\bar{\omega}_{0}=0.815 \omega_{0}\right)$ ac-field $s \sin \Omega t$ as obtained from the smallest nonzero eigenvalue of the rate matrix (9). The three asterisks $*$ denote findings of an exponential fit to the QUAPI results. Note the resonant enhancement at finite driving strength as the temperature is lowered $\left(T_{0}=\hbar \omega_{0} / k_{B}\right)$. Here and in the inset we set $E_{\mathrm{B}}=1.4 \hbar \omega_{0}, \gamma=0.1 \omega_{0}$, $\omega_{c}=10 \omega_{0}$. Inset: Evolution of the population of the left well as predicted by the GME (6) with (7), by its Markovian approximation and by the quasi-adiabatic path integral method (QUAPI). We choose $T=0.1 T_{0}, s=1.0 s_{0}$.

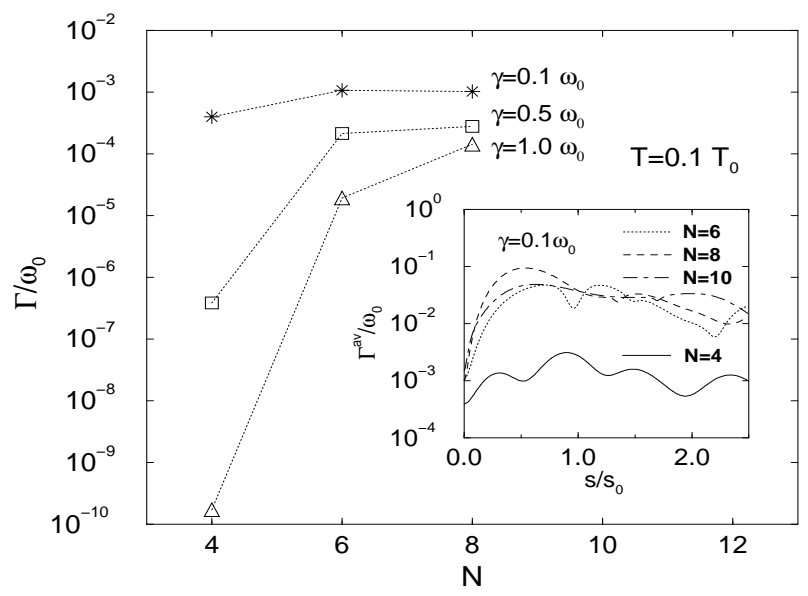

FIG. 3. Static deacy rate $\Gamma$ vs. the number $N$ of levels used to truncate the full double-well dynamics. Even at moderate temperatures $T=0.1 T_{0}$ the reduction to the two lowest doublets is appropriate for moderate damping. Here and in the inset we set $E_{\mathrm{B}}=1.4 \hbar \omega_{0}, \omega_{c}=10 \omega_{0}$. Inset: Averaged transfer rate $\Gamma^{\text {av }}$ vs. driving strengths $s$ of a resonant field $\left(\Omega=\bar{\omega}_{0}\right)$ at $T=0.1 T_{0}$. Convergence requires now $N>4$. 